\title{
An Eliminating Brightness Differences Algorithm for Lunar Surface Image
}

\author{
Hou Ying ${ }^{1, a}$, Wang Guicai ${ }^{2, b}$ \\ ${ }^{1}$ Zhengzhou Normal University, ZhengZhou, 450044, China \\ ${ }^{2}$ HeNan University of Technology, ZhengZhou, 450001, China \\ aemail: houyin69@163.com, bemail: wangguicai@tom.com
}

Keywords: Image enhancement; Color components; Relative constraint; Lunar surface image

\begin{abstract}
For solving effect and real-time performance problems of lunar surface image, the paper proposed an eliminating brightness differences algorithm of lunar surface image by correlation of color components fitting gray level map function. Feature points were obtained by matching in process image, which could establish normalized RGB vector space. The max-relativity feature points were chosen by correlation of color components and random sample consensus algorithm. The gray level map function was fitted by RANSAC and matching feature points, which could eliminate different brightness between two images. The experimental results show that this method could not only eliminate different brightness between lunar surface images, but also meet real-time processing requirement of lunar rover.
\end{abstract}

\section{Introduction}

The moon not only has rich resources but also was an important base of explore other distant planets, thus world powers once again had taken off a upsurge Lunar Exploration and made lunar projects [1] in recent years. When the vision system parameters of lunar rover could not be completely consistent image acquisition may result in the presence of lunar surface brightness differences; another lunar unstructured environments uneven illumination characteristics may also lead to a strong light on one side, the other side light intensity was weak, which could have more brightness difference in the lunar surface image [2]. Currently researchers have done a lot in terms of image enhancement research, but these methods and more structured environment based on the lunar surface cannot meet their basic requirements unstructured environment of lunar rover [3,4]: real-time image fusion problem, while environmental noise has strong robustness. Due to Lunar surface environment was natural (irregular, unstructured and strong light intensity) environment, the grayscale of Lunar surface image change was not obvious grayscale, dull coloring. In additional, there were not life activity and motion feature on the lunar surface, which the lunar surface image was usually gray image [5]. In view of the above analysis, this paper presents a practical and effective elimination of the lunar surface brightness of the image difference algorithm.

\section{Algorithm Description}

The algorithm step was shown in figure 1. 1) sample lunar surface image with brightness differences; 2) feature point extraction and matching; 3) set up the normalized RGB model using the grayscale of feature matching point extraction in processing image; 4) eliminate feature matching points with poor color correlation by color components correlation constraint in target image; 5) calculate level map function parameters using feature matching points with strong color correlation in target image; 6) eliminate the brightness difference with level map function for Lunar Surface Image.

\begin{tabular}{|c|c|c|c|c|c|}
\hline $\begin{array}{l}\text { the lunar surface image with } \\
\text { brightness difference }\end{array}$ & \begin{tabular}{|c|} 
feature point extraction \\
and matching
\end{tabular} & set up the normalized & $\begin{array}{c}\text { color components } \\
\text { correlation constraint }\end{array}$ & $\begin{array}{l}\text { calculate level map } \\
\text { function parameters }\end{array}$ & $\begin{array}{c}\text { eliminate the } \\
\text { brightness difference }\end{array}$ \\
\hline
\end{tabular}

Fig.1 Eliminating brightness differences algorithm step for lunar surface image 


\section{SIFT Features Point Extraction and Matching}

The paper obtained believable feature matching points by SIFT [6].The SIFT features point extraction and matching has five major stages:

Step 1: Scale-space extreme detection. $D(x, y, \sigma)$ was the difference scale space of image. $G(x, y, \sigma)$ was the convolution of a variable-scale Gaussian, $I(x, y)$ was the input image.

$$
\begin{aligned}
& D(x, y, \sigma)=[G(x, y, k \sigma)-G(x, y, \sigma)] \times I(x, y)=\mathrm{L}(x, y, k \sigma)-\mathrm{L}(x, y, \sigma) \\
& G(x, y, \sigma)=e^{-\left(x^{2}+y^{2}\right) / 2 \sigma^{2}} / 2 \pi
\end{aligned}
$$

Where $\times$ was the convolution operation in $\mathrm{x}$ and $\mathrm{y}$. $\mathrm{L}$ was the Gaussian image.

Step2: Keypoint localization. Keypoint could be located subpixel position using the quadratic Taylor expansion $D(x, y, \sigma)$ in scale-space. This Taylor expansion was given by

$$
D(x)=D+\left(\partial D^{T} / \partial x\right) * x+x^{T} *\left(\partial^{2} D /\left(2 * \partial x^{2}\right)\right) * x
$$

Where $D$ and its derivatives were evaluated at the candidate keypoint. $x=(x, y, \sigma)$ denoted the offset from this point.

Step3: Orientation assignment, the amplitude and phase angle of gradient was carried out as below Eq. 5 and Eq. 6:

$$
\begin{aligned}
& M=\sqrt{\left(L_{1}-L_{4}\right)^{2}+\left(L_{2}-L_{3}\right)^{2}}, \theta(x, y)=\arctan \left(\left(L_{1}-L_{4}\right) /\left(L_{2}-L_{3}\right)\right) \\
& L_{1}=L(x+1, y), L_{2}=L(x, y+1), L_{3}=L(x, y-1), L_{4}=L(x-1, y)
\end{aligned}
$$

Where $m(x, y)$ denoted amplitude of gradient. $\theta(x, y)$ denoted phase angle of gradient. We divided phase angle into 36 portions. the maximal direction was the key-point's direction.

Step4: Keypoint descriptor. taking any features point as the center of $8 \times 8$ neighborhood, this neighborhood was evenly divided into $2 \times 2$ sub-region (the size of each sub-region was $4 \times 4$ ), The descriptor $(2 \times 2 \times 8$ dimensional vector) could be obtained.

Step5: Features point matching. For SIFT features matching points of two images, because of the images had been epipolar rectification before matching, corresponding points had only the horizontal offset, if $\left|m-m^{\prime}\right| \leq \varepsilon, m$ and $m^{\prime}$ was a pair corresponding point, otherwise, $m$ and $m^{\prime}$ was not a pair corresponding point [2].

\section{Normalized Model}

Most of natural colors were composed of Red, Green and Blue in term of the different proportion. Meanwhile, the majority of monochromatic light could be also decomposed into Red, Green, Blue color light. It was tricolor theory in colorimetric. Its mathematical description was often RGB vector space.

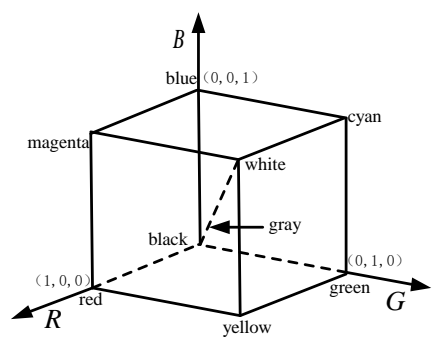

Fig.2 RGB vector space

RGB vector space was not only the most typical but also the most common color models based on the Cartesian coordinate system. Is three axes respectively represent the $R, G, B$ three color components. Black corresponded to the origin, the farthest vertex from the origin corresponded to white. The distribution of gray value was the main diagonal from black to white. The other space points corresponded to different colors. All gray represented by the linear combinations of $R, G, B$ vectors and also by the vector between origin and this point (as shown in Figure 2). The actual RGB color cube was often normalized so that the distribution ranges of $R, G, B$ color components were 
$0 \sim 1$. The gray value of ideal image was the main diagonal distributed0 in the RGB vector space. Due to environmental noise, the gray value of lunar surface images distributed in two sides of main diagonal. In the light of this, the paper firstly constructed RGB vector space via the feature point. Its gray values were assigned to the $R, G, B$ color components; then space points were constructed with color components. Finally the max-relativity feature points were chosen by correlation of color components and RANSAC (Random Sample Consensus) method.

Fishler and Bolles proposed RANSAC method which was very effective for a large proportion outliers [7,8]. The SIFT feature matching points of lunar surface images $m, m$ ' were used to correlation constraint of color components and automatically calculate constraint line $l$, which could guide constraint to obtain the SIFT feature matching points with more stronger correlation $m, m$. Its algorithm structure described as follows:

\section{Objective}

The paper used the SIFT feature matching points $m, m$ ' to estimate correlation constraint line of color components by RANSAC method, and the SIFT feature matching points with stronger correlation $m, m^{\prime}$ were extracted. Pay Attention to $\hat{m} \in m, \hat{m}^{\prime} \in m^{\prime}$. The main steps were as follows:

\section{Algorithm}

Step 1:(SIFT matching points): (SIFT matching points): Choose SIFT feature matching points with partial brightness (assumed $m$ ) among $m$ and $m$ of lunar surface image.

Step 2(RANSAC robust estimation): Repeat for $N$ samples, where $n$ is determined adaptively as in [7]:

Select a random sample of 2 SIFT feature matching points and compute constraint line $l$ from SIFT feature matching points $m$. It gets a solution;

Calculate the distance $d_{\perp}$ for each putative SIFT feature matching point;

Compute the number of inliers consistent with $l$ by the number of correspondences for which $d_{\perp}<t$ pixels. Where $t$ was distance threshold;

If there are real solutions for $l$ the number of inliers was computed for each solution, and the solution with most inliers retained.

Step 3(Non-linear estimation): Re-estimate $l$ from all SIFT matching points classified as inliers by minimizing a cost function, using the Levenberg-Marquardt algorithm.

Step4: Compute fundamental matrix $F$ between two images by SIFT matching points (The normalized 8-point algorithm)

Step5: Calculation accuracy of fundamental matrix $F$ directly determines matching effect. This paper uses RANSAC to automatic estimate $F$ [7].

\section{Algorithm Implementation and Experimental Results}

The Experimental lunar surface images AS17 AS17-21495-21495 (as shown in figure 3 (a-b)) were provided by National Aeronautics and Space Administration (NASA). in order to more effectively assess my algorithm's brightness difference eliminate effect, the paper enhanced image AS17-21494 brightness to simulate brightness difference due to un-uniform light under lunar surface unstructured environment and the cameras of lunar rover hardware performance inconsistent. Enhanced brightness image was AS17-21494-1 (as shown in figure 3 (c)). it was not difficult to see that there were clear large brightness differences between the image AS17-21494 and AS17-21494-1 Meanwhile, in order to increase the algorithm comparability, this paper are compared and experimental verification for literature [8] (the Nemra method). 


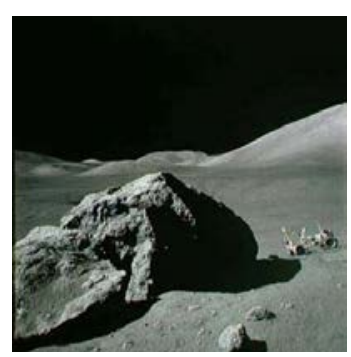

(a) Image AS17-21495

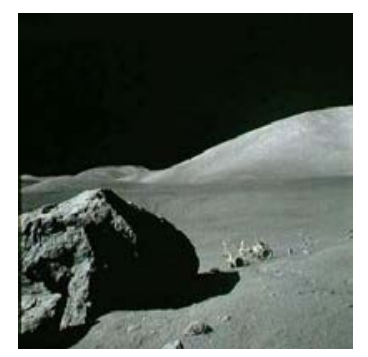

(b) Image AS17-140-21494

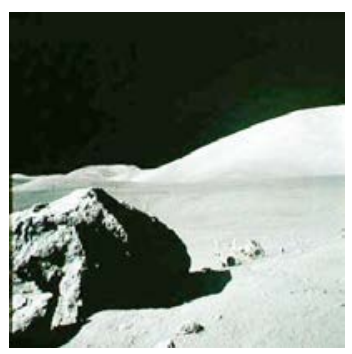

(c) Image AS17-140-21494-1

Fig.3 Lunar surface image

First, the joint constraint results were shown in FIG 4 (a) by color components correlation and RANSAC in RGB vector space. The experimental results used the two-points to express fitting straight line (the starting point denoted by $l_{\text {start }}$. the endpoint denoted by $l_{\text {end }}$ ). The fitting result was as follows $l_{\text {start }}=\left(\begin{array}{lll}0.043 & 0.031 & 0.075\end{array}\right), l_{\text {end }}=\left(\begin{array}{lll}0.894 & 0.875 & 0.915\end{array}\right)$. The Fig 4 (b-d) showed the respectively fitting straight line projection in $\mathrm{RG}, \mathrm{RB}$ and $\mathrm{BG}$ three planes. It was obviously seen that the fitting straight line had the greatest consistent in three planes. In addition, it retained the most strong feature point for color component relevant.

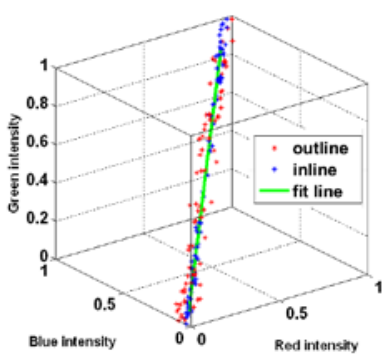

(a)RGB vector space

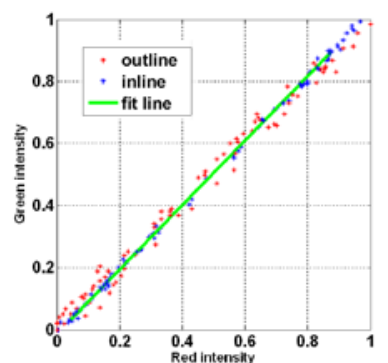

(b) RG vector space

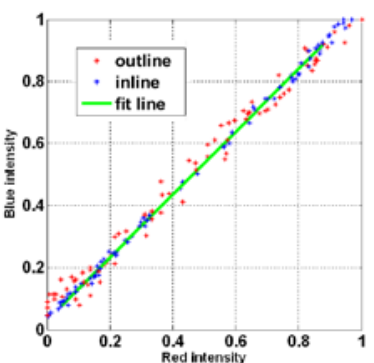

(c) RB vector space

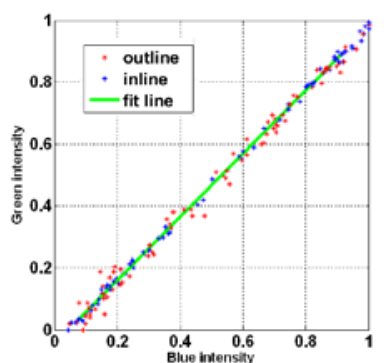

(d) BG vector space

Fig.4 linear fitting Results of color components correlation constraint

Secondly, by analyzing the characteristics of gray-scale data matching points between the two images (Figure 5) shows that the direct method using Nemra fitting gray mapping function there was a big error; through this method for mapping function to be gray together to get the best fit results.

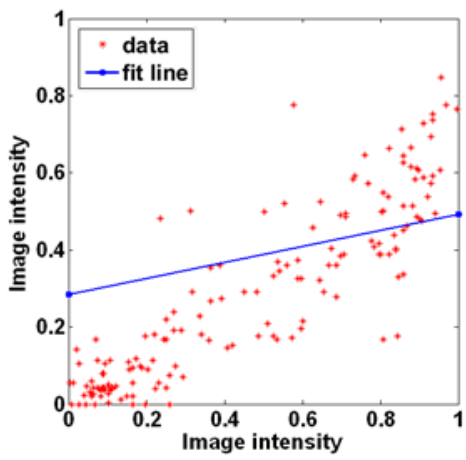

(a)Nemra mehod

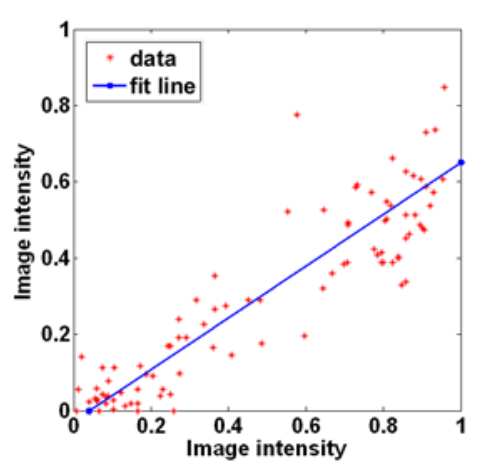

(b) The suggested method

Fig.5 Fitted results of different gray level map functions

Again, the quantitative evaluation index of Table 1 showed that the fitting parameters $c, d$ of Nemra method were serious distortion compared with the suggested method. The suggested method executed twice RANSAC algorithm lead to increase $0.267 \mathrm{~s}$ than Nemra method in run time. 
Table 1 Fitted results of different gray level map function

\begin{tabular}{ccc}
\hline Evaluation Index & The suggested method & Nemra method \\
\hline fitting parameter $c$ & 0.6770 & 0.1932 \\
fitting parameters $d$ & 0.0271 & 0.2664 \\
computation time $(/ \mathrm{s})$ & $0.249+0.102$ & 0.084 \\
\hline
\end{tabular}

Finally, differences in brightness elimination results in Figure 6 see, compared to Nemra method, The suggested method has small color distortion and significantly improved subjective visual effect on the image.

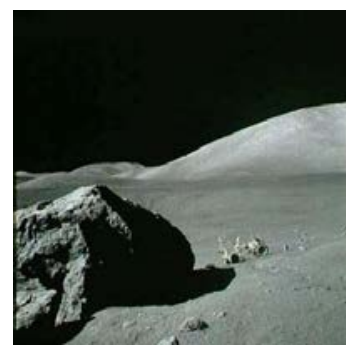

(a) Image AS17-21494

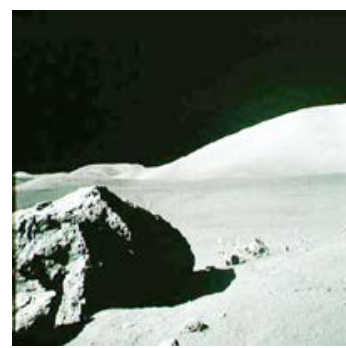

(b) Image AS17-21494-1

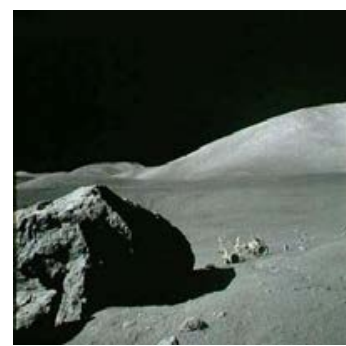

1 (c) The suggested method

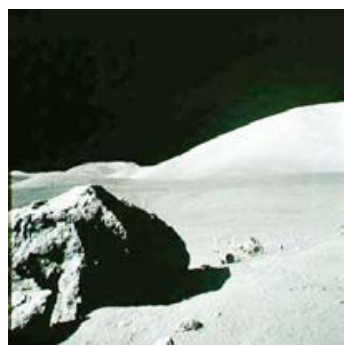

(d) Nemra method

Fig.6 Comparison results of lunar surface image brightness eliminating

\section{Conclusion}

The paper took the lunar surface environment and its basic requirements of lunar rover as the starting point, used correlation constraint of color components and RANSAC algorithm to fit gray level map function, and a fast eliminate brightness differences algorithm for the lunar surface image was proposed. The experimental results show that this algorithm has the advantages of significantly improve the effect of brightness differences eliminate, small color distortion and high real-time processing requirement. All these show the lunar rover could really adapt to the lunar surface unstructured environment.

\section{Acknowledgement}

This work was supported by National High-tech Research and Development Program of China(863 Program) under Grant 2012AA101008, the Doctoral Foundation of Henan University of Technology under Grant 2012BS062 and the Basic Research Development Program of Education Department Henan Province under Grant 13A520171.

\section{References}

[1] Liu Jian-zhong, Ouyang Zi-yuan, Liu Chun-lai et al. China National Moon Exploration Progress(2001-2010)[J], Bulletin of Mineralogy, Petrology and Geochemistry, 32(5),2013. 544-551.

[2] Szeliski R. Image alignment and stitching: a tutorial [R]. Microsoft Research of Microsoft Corporation. 2006.

[3] Powell, M W, Norris J S, Vona M A, et al. Scientific visualization for the mars exploration rovers [C]. The IEEE International Conference on Robotics and Au-tomation (ICRA'05), Barcelona, Spain. 2005, 4290-4296.

[4] Bentoutou Y, Taleb N, Kpalma K, et al. An automatic image registration for applications in remote sensing [J]. IEEE Transactions on Geosciences and Remote Sensing.,2005, 43(9): 2127-2137.

[5] Yang A Y, Hong W, Ma Y. Structure and pose from single images of symmetric objects with 
applications to robot navigation [C]. The IEEE, International Con-ference on Robotics \& Automation, Taipei. 2003, 1013-1020.

[6] Lowe D G. Distinctive image features from scale-invariant keypoints[J]. International Journal of Computer Vision. 2004, 60(2): 91-110.

[7] Fishier M A, Boles RC. Random Sample Concensus: A Paradigm for Model Fitting with Applications to Image Analysis and Automated Cartography[J]. Communications of ACM. 1981, 24(6): 381-395.

[8] Nemra A, Aouf N, Ecole M P, et al. Robust invariant automatic image mosaicing and super resolution for uav mapping[C]. The 6th International Symposium on Mechatronics and Its Applications (ISMA’09), Sharjah, United Arab Emirates. 2009, 1-7. 